\title{
BMJ Open Diarrhoea: interventions, consequences and epidemiology in the intensive care unit (DICE-ICU): a protocol for a prospective multicentre cohort study
}

\author{
Joanna C Dionne, ${ }^{\bullet 1,2,3,4}$ Kristen Sullivan, ${ }^{3}$ Lawrence Mbuagbaw, ${ }^{\oplus 1}$ \\ Alyson Takaoka, ${ }^{1}$ Erick Huaileigh Duan, ${ }^{1,3,4,5}$ Waleed Alhazzani, ${ }^{1,3,4}$ John W Devlin, ${ }^{6}$ \\ Matthew Duprey, ${ }^{6}$ Paul Moayyedi, ${ }^{2,3,7}$ David Armstrong, ${ }^{2,3,7}$ Lehana Thabane, ${ }^{1}$ \\ Jennifer L Y Tsang, ${ }^{\oplus, 5}$ Roman Jaeschke, ${ }^{1,3,4}$ Cindy Hamielec, ${ }^{3,4}$ Tim Karachi, ${ }^{3,4}$ \\ Rodrigo Cartin-Ceba, ${ }^{8}$ John Muscedere, ${ }^{9,10}$ \\ Mohammed Saeed Saad Alshahrani, ${ }^{11}$ Deborah J Cook ${ }^{1,3,4}$
}

To cite: Dionne JC, Sullivan K, Mbuagbaw L, et al. Diarrhoea: interventions, consequences and epidemiology in the intensive care unit (DICE-ICU): a protocol for a prospective multicentre cohort study. BMJ Open 2019;9:e028237. doi:10.1136/ bmjopen-2018-028237

- Prepublication history and additional material for this paper are available online. To view please visit the journal (http:// dx.doi.org/10.1136/bmjopen2018-028237).

Received 28 November 2018 Revised 3 April 2019 Accepted 21 May 2019

Check for updates

(c) Author(s) (or their employer(s)) 2019. Re-use permitted under CC BY-NC. No commercial re-use. See rights and permissions. Published by BMJ.

For numbered affiliations see end of article.

Correspondence to Dr Joanna C Dionne; joanna.dionne@medportal.ca

\section{ABSTRACT}

Introduction Diarrhoea is a frequent concern in the intensive care unit (ICU) and is associated with prolonged mechanical ventilation, increased length of ICU stay, skin breakdown and renal dysfunction. However, its prevalence, aetiology and prognosis in the critically ill have been poorly studied. The primary objectives of this study are to determine the incidence, risk factors and consequences of diarrhoea in critically ill adults. The secondary objectives are to estimate the incidence of Clostridium difficileassociated diarrhoea (CDAD) in ICU patients and to validate the Bristol Stool Chart and Bliss Stool Classification System characterising bowel movements in the ICU. Our primary outcome is the incidence of diarrhoea. Our secondary outcomes include: CDAD, ICU and hospital mortality and ICU and hospital length of stay.

Methods and analysis This international prospective cohort study will enrol patients over 10 weeks in 12 ICUs in Canada, the USA, Poland and Saudi Arabia. We will include all patients 18 years of age and older who are admitted to the ICU for at least 24 hours and follow them daily until ICU discharge. Our primary outcome is the incidence of diarrhoea based on the WHO definition, during the ICU stay. Our secondary outcomes include: CDAD, ICU and hospital mortality and ICU and hospital length of stay. We will use logistic regression to identify factors associated with diarrhoea (as defined using WHO criteria) and the kappa statistic to measure agreement on diarrhoea rates between the WHO definition and the Bristol Stool Chart and Bliss Stool Classification System.

Ethics and dissemination The protocol has been approved by the research ethics board of all participating centres. The diarrhoea interventions, consequences and epidemiology in the intensive care unit (DICE-ICU) study will generate evidence about diarrhoea and its frequency, predisposing factors and consequences, to inform critical care practice and future research.

Lay summary Diarrhoea is a frequent clinical problem for hospitalised patients including those who are critically ill in the ICU. Diarrhoea can cause complications such as skin damage, dehydration and kidney problems. It is not
Strengths and limitations of this study

- Large prospective, international, multicentre, cohort study of a mixed population of critically ill adults.

- Comprehensive evaluation of diarrhoea incidence and its potential risk factors throughout the intensive care unit stay.

- Bedside nurse characterisation of all bowel movements with the WHO definition, Bristol Stool Chart and Bliss Stool Classification System to validate these scoring tools in critically ill adults.

- Possible missing data to characterise some bowel movements.

- Possible reporting bias or observer bias influencing some data collection.

clear how common diarrhoea is in the ICU, the factors that cause it or the best way for clinicians to assess it. The DICE-ICU study is an international prospective observational study to examine the frequency, risk factors and outcomes of diarrhoea during critical illness.

\section{INTRODUCTION}

The reported incidence of diarrhoea among critically ill patients ranges from $2 \%$ to $95 \% .^{12}$ This wide range is due to the lack of a universally accepted definition in the intensive care unit (ICU). It is often difficult to differentiate true diarrhoea (the passage of more than three liquid bowel movements per 24 hours) from a change in stool frequency or stool consistency (eg, looser stools). There is also wide variation in what is considered 'a normal bowel habit', ${ }^{3}$ ranging from two to three bowel movements per day to three bowel movements per week. Such 'normal variation' makes it challenging to define diarrhoea and 
to identify what may be 'abnormal' in the ICU setting. The concept of what constitutes a normal bowel pattern in the ICU has not been well studied. The ideal definition of diarrhoea in the ICU remains unclear.

The WHO defines diarrhoea as the passage of three or more liquid stools per day. While simple, and easily applied at the bedside, clinicians rarely refer to this definition in ICU practice. A criticism of the WHO definition is that quantification of stool is not necessarily an accurate indicator of colonic transit time. The most recognised stool evaluation instrument in hospitals is the Bristol Stool, ${ }^{5}$ comprising seven categories with a graphical depictions and text descriptions for each category. A Bristol Score of 6 or 7 is classified as diarrhoea. ${ }^{6}$ The Bristol Stool Chart is a better predictor of whole-intestinal transit time than stool frequency. ${ }^{5}$ The Bristol Stool Chart has subsequently been used to define diarrhoea by the European Society for Clinical Microbiology and Infectious Disease, and has used the Bristol Stool Chart to define diarrhoea for Clostridium difficile infection. ${ }^{7}$

The Bliss Stool Classification System is an alternative system initially developed to assess stool consistency in patients with faecal incontinence. The tool has four categories with depictions and descriptions and can be applied at the bedside, but with fewer categories; it has a good reliability when used by healthcare professionals, nursing students and volunteers. ${ }^{8}$ Further reliability and validity testing has been performed, ${ }^{89}$ though this instrument has not been as widely used in research. There are no studies that validate the Bristol Stool Chart or the Bliss Stool Classification System in the ICU setting for either clinical or research purposes.

Antibiotics, antifungal therapy, prokinetics and enteral nutrition may predispose to diarrhoea in the critically $\mathrm{ill}^{2}$; however, the risk of diarrhoea associated with these factors is unclear and poorly quantified due to the retrospective designs and small sample sizes of previous studies. Without strong evidence informing ICU clinicians of the possible aetiologies of diarrhoea, enteral nutrition is often considered the culprit, and feeds are discontinued. ${ }^{1011}$ While the enteral route is the preferred method of nutrition delivery in the ICU, ${ }^{12}$ if diarrhoea is misattributed to enteral nutrition, unnecessary feeding interruption may exacerbate caloric and protein deficits.

Studies on the epidemiology of diarrhoea in critically ill patients are limited. These studies have explored issues of gastrointestinal failure (eg, feeding intolerance, gastrointestinal haemorrhage and ileus), ${ }^{13}$ diarrhoea in enterally fed critically ill patients ${ }^{10}$ or risk factors of diarrhoea. ${ }^{14}{ }^{15}$ Research designs to date have included database registry studies ${ }^{15}$ case-control studies ${ }^{14}$ and retrospective audits. Interest in diarrhoea has become particularly relevant as enteral nutrition, often considered the cause of diarrhoea in the ICU, is used earlier and more often than in the past. Furthermore, there is growing concern about Clostridium difficile-associated diarrhoea (CDAD) in this setting.

We are conducting a prospective multicentre study with the following objectives: to determine the incidence and frequency of diarrhoea, risk factors for diarrhoea and consequences (ICU and hospital mortality, ICU and hospital length of stay) of diarrhoea in critically ill adults and validate different stool classification systems. The primary outcomes are to determine the frequency and the incidence of diarrhoea, defined using the WHO criteria and risk factors for diarrhoea in this patient population. The secondary outcomes are to estimate the incidence of CDAD in ICU patients, validate the Bristol Stool Chart and Bliss Stool Classification System for characterising bowel movements, ICU and hospital mortality and ICU and hospital length of stay.

\section{METHODS AND ANALYSIS \\ Design}

The diarrhoea interventions, consequences and epidemiology in the intensive care unit (DICE-ICU) study is a 10 week prospective cohort study of consecutively admitted critically ill patients, and will be conducted at 12 academic and community medical and surgical ICUs in; Canada, the USA, Poland and Saudi Arabia.

\section{Participants}

We will include all consecutive patients 18 years of age or older admitted to the ICU for at least 24 hours, regardless of their mechanical ventilation status. There are no exclusion criteria except patients admitted to the ICU for $<24$ hours. At centres with multiple ICUs, we will enrol patients in medical, surgical and mixed ICUs rather than specialised ICUs (eg, cardiovascular surgery units). In each participating ICU, we will document several centrelevel variables including the number of ICU and hospital beds, population case-mix, unit design, university affiliation and use of a 'bowel protocol' (an established order set of prescribed laxatives and/or motility agents with parameters that describe when to use these medications for patients who have not had a bowel movement). ${ }^{16}$

\section{Patient and public involvement}

Our protocol did not have a patient or patient family member engagement in its development of the research question, ascertainment of outcomes or methodology. Our population of interest is critically ill patients who are either mechanically ventilated, comatose or have altered level of consciousness due to their underlying critical illness or associated sedation. Such patient characteristics which typically persist for the majority of their ICU admission preclude meaningful real-time patient engagement as the study progresses. However, our ethics review board includes patient representatives who provided input to the design of the protocol and its implementation. Also, we will disseminate the results of the study to patients, families and citizens through multimedia methods including pamphlets, social media and research boards in the ICU setting.

\section{Enrolment}

Daily, research coordinators will screen all newly admitted patients to each participating ICU who will also document 
the prior location (eg, emergency department, operating room, medical or surgical ward), hospital and ICU admission dates. The research coordinators will collect baseline patient characteristics including age, sex and chronic comorbidities (pre-hospital), and Acute Physiology and Chronic Health Evaluation II (APACHE II) score and admitting diagnosis (at ICU admission). Conditions associated with an increased risk for diarrhoea (eg, gastrointestinal bleeding, history of short bowel syndrome, inflammatory bowel disease, history of Clostridium difficile infection and the presence of ileostomy or colostomy) or a decreased risk of diarrhoea (eg, opiates) (pre-ICU period) will also be documented.

\section{Outcomes}

We will perform prospective daily data collection until death in the ICU or ICU discharge. Daily, the bedside nurse will use the case report form refined during the DICE pilot study ${ }^{17}$ to track all stools. The number and character of each stool will be documented daily using the WHO definition and the Bristol Stool Chart and Bliss Stool Classification System. We will use these data to ascertain our primary outcome of the incidence and frequency of diarrhoea in the study population over a 10 week period in participating ICUs.

Research coordinators will collect data daily, completing a standardised, previously piloted and refined case report form (CRF) ${ }^{17}$ The CRF (online supplementary appendix 1) includes data on: life support utilisation (mechanical ventilation, vasopressor usage, renal replacement therapy), laboratory values, physiotherapy and clinical outcomes. Research coordinators will also document whether nutrition was administered, nutrition formulation, administrative route (enteral or parenteral), infusion rate and any feeding interruption that may be risk factors for diarrhoea (primary outcome). We will document nutritional targets as determined by the ICU dietitians and whether target rates are met. Research coordinators or bedside pharmacists will also track relevant medications (eg, antibiotics, acid suppressants, antifungal agents, prokinetics, opioids, laxatives and hyperosmolar medications) that may be risk factors for diarrhoea (primary outcome). Research coordinators will document the consequences of diarrhoea such as electrolyte abnormalities, use of anti-diarrhoeal agents, use of faecal management devices and diagnostic test ordering (eg, for C. difficile, malabsorption, etc). Research coordinators will collect detailed data on all patients developing CDAD (secondary outcome). We will also document the length of ICU (secondary outcome) and hospital stay (secondary outcome) and mortality (ICU and hospital) on all patients enrolled in the study (secondary outcomes).

\section{Data management}

The research coordinator at each site will enter the data locally into a web-based system (iDatafax, V.4.3.0, 2013). ${ }^{18}$ A data manager at the McMaster University DICE-ICU Methods Centre will validate all data, ensuring that ambiguous, out of range or missing data are identified and addressed in a timely manner. We will make every attempt to resolve missing data by querying participating centres. If data remain missing, we will address this with the multiple imputation methods, based on the type and distribution of missing data.

\section{Training of sites}

Research coordinators at each site will be oriented by the principal investigator to the data collection forms though site initiation visits in-person or by webinar, and standard operating procedures. At each site, bedside nurses will be oriented to the stool classification systems and trained by the research coordinator on how to record the patient's bowel movements on the case report forms at scheduled sessions and at the bedside. Throughout the study, the methods centre data manager will also give suggestions and feedback to the site research coordinators on data collection to ensure protocol fidelity and uniformity across sites.

\section{Central adjudication}

In duplicate, two independent adjudicators will review all possible cases of CDAD. Patients who have a possible $C$. difficile infection will be adjudicated using the Infectious Disease Society of America criteria. ${ }^{19}$ For all possible cases, the following will also be adjudicated: stool frequency, complications (eg, colectomy), treatments (antibiotics, surgery) and overall severity according to guidelines of the European Society of Clinical Microbiology and Infectious Diseases, ${ }^{20}$ Infectious Disease Society of America ${ }^{19}$ and American College of Gastroenterology. ${ }^{21}$

\section{Sample size}

Our sample size estimation is based on two approaches. The first is the standard rule of thumb approach which is based on the independent factors under examination (in our four-centre DICE pilot study, there were eight independent risk factors for diarrhoea) and the number of events required for each degree of freedom (df) which requires 20 events per factor. Using this approach, with eight independent risk factors and seven df, we would require 140 patients with diarrhoea to examine these factors in a multivariate analysis. ${ }^{22}$ Our second approach derives the sample size based on our DICE pilot study with the primary objective of determining independent factors associated with diarrhoea during critical illness. We have used the results of our DICE pilot study in which antibiotic exposure (main independent variable) was associated with diarrhoea (adjusted OR 2.15, 95\% CI 1.04 to 4.4) in the logistic regression to justify the sample size. Our sample size is computed for the research question that would require the largest sample size (which is the diarrhoea risk factor analysis), inherently providing sufficient power to address the other objectives. In the DICE pilot study, we included the following variables: age, sex, APACHE II score and use of relevant drugs (eg, motility modifiers, stool softeners). Among the 268 patients $(80 \%$ 
Table 1 Statistical analysis plan

\begin{tabular}{|c|c|c|c|}
\hline Research objectives & Outcome & Covariates & Analytical approach \\
\hline \multirow{5}{*}{$\begin{array}{l}\text { To determine incidence of } \\
\text { diarrhoea and consistency } \\
\text { of bowel movements during } \\
\text { critical illness } \\
\text { To determine the frequency } \\
\text { of diarrhoea defined as the } \\
\text { proportion of patients with new } \\
\text { ICU-acquired diarrhoea }\end{array}$} & Primary outcome & \multirow[t]{5}{*}{ NA } & \multirow{5}{*}{$\begin{array}{l}\text { The incidence of diarrhoea will } \\
\text { be computed as the number } \\
\text { of new cases during ICU stay } \\
\text { divided by the person-time at } \\
\text { risk. } \\
\text { Descriptive statistics } \\
\text { (proportion of patients with } \\
\text { diarrhoea) and corresponding } \\
95 \% \mathrm{Cl}\end{array}$} \\
\hline & WHO-defined diarrhoea & & \\
\hline & $\begin{array}{l}\text { Different definitions of } \\
\text { primary outcome }\end{array}$ & & \\
\hline & $\begin{array}{l}\text { Bristol Stool Chart-defined } \\
\text { diarrhoea }\end{array}$ & & \\
\hline & $\begin{array}{l}\text { Bliss Stool Classification } \\
\text { System-defined diarrhoea }\end{array}$ & & \\
\hline \multirow{3}{*}{$\begin{array}{l}\text { To determine risk factors } \\
\text { associated with diarrhoea } \\
\text { during critical illness }\end{array}$} & Primary outcome & \multirow{3}{*}{$\begin{array}{l}\text { Age, sex, APACHE II score, } \\
\text { drugs (motility modifiers, } \\
\text { opiates, stool softeners), prior } \\
\text { gastrointestinal disease, centre }\end{array}$} & \multirow{3}{*}{ Logistic regression } \\
\hline & Dependent variable & & \\
\hline & WHO-defined diarrhoea & & \\
\hline \multicolumn{4}{|l|}{ Secondary outcomes } \\
\hline Research objective & Secondary outcome & Covariates & Analytic approach \\
\hline \multirow{4}{*}{$\begin{array}{l}\text { To determine the } \\
\text { consequences of diarrhoea }\end{array}$} & Dependent variable & \multirow{4}{*}{$\begin{array}{l}\text { WHO-defined diarrhoea, age, } \\
\text { sex, APACHE II } \\
\text { score }\end{array}$} & \multirow[t]{3}{*}{ Cox regression } \\
\hline & Time to ICU discharge & & \\
\hline & Time to hospital discharge & & \\
\hline & Mortality & & Logistic regression \\
\hline Research objective & Secondary outcome & Covariates & Analytical approach \\
\hline \multirow{3}{*}{$\begin{array}{l}\text { To determine the incidence of } \\
\text { Clostridium difficile-associated } \\
\text { diarrhoea (CDAD) during } \\
\text { critical illness }\end{array}$} & IDSA-defined CDAD & \multirow[t]{3}{*}{ NA } & \multirow{3}{*}{$\begin{array}{l}\text { Descriptive statistics } \\
\text { (proportion of cases with } \\
\text { diarrhoea during study period) }\end{array}$} \\
\hline & ESCMID-defined CDAD & & \\
\hline & ACG-defined CDAD & & \\
\hline $\begin{array}{l}\text { To determine agreement } \\
\text { between WHO and } \\
\text { Bristol and Bliss scores }\end{array}$ & $\begin{array}{l}\text { Chance corrected agreement } \\
\text { (kappa score) }\end{array}$ & NA & Kappa statistic with $95 \% \mathrm{Cl}$ \\
\hline
\end{tabular}

APACHE II, Acute Physiology and Chronic Health Evaluation II; ACG, American College of Gastroenterology ; ESCMID, European Society of Clinical Microbiology and Infectious Diseases ; ICU, intensive care unit; IDSA, Infectious Disease Society of America ; NA, not applicable; CDAD, Clostridium-difficile associated diarrhoea.

of total population) exposed to antimicrobials, $182(67 \%)$ had diarrhoea. Among the 67 patients not exposed to antimicrobials, only 19 (28\%) had diarrhoea (crude OR of $5.34,95 \%$ CI 2.96 to 9.64 ). We have computed sample sizes for a range of plausible effect sizes (based on CIs from the DICE pilot study). Approximately 1000 patients are required to detect an OR as small as 1.6 at level of significance $\alpha=0.05$ and power $(1-\beta)=0.8$, if $80 \%$ of the total population is exposed to antimicrobials (computations by G Power V.3.1.9.2) ${ }^{23}$ Given the consideration of both approaches, this study will be adequately powered to answer our primary research questions and adequately explore risk factors for diarrhoea. Given the observational design, the ultimate sample size will be determined by the number of patients admitted to the ICU in participating centres during the study period; we will target at least 1000 critically ill adults.

\section{Data analysis}

Analysis will include descriptive and inferential statistics. We present our detailed statistical plan for evaluation of the primary and secondary outcomes in table 1 . We will report baseline characteristics, that will be described using counts (percentage) for categorical variables and mean (SD) or median (IQR) for continuous variables as appropriate. Our primary outcome will be defined by WHO criteria of $\geq 3$ liquid bowel movements/day, and additional definitions of diarrhoea including a Bristol Stool Chart score of 6 or 7, and Bliss Stool Classification System score of 4 will also be reported. The incidence of diarrhoea will be computed as the number of new cases during ICU stay divided by the person-time at risk. We will also report the frequency of diarrhoea defined as the proportion (the number of patients who have developed diarrhoea/the number of patients within the DICE-ICU cohort) of patients with new ICU-acquired diarrhoea and will be computed for all three definitions.

The role of covariates (eg, age, sex, APACHE II score, drugs, prior gastrointestinal disease) in the occurrence of diarrhoea will be analysed with a logistic regression model that we developed during the DICE pilot study. ${ }^{17}$ The consequences of diarrhoea (time to ICU and time to hospital discharge) will be described and assessed using Cox regression; the influence of diarrhoea on mortality will be assessed using logistic regression techniques. In 
these models, covariates will be entered as a block. Goodness-of-fit will be assessed by examining the residuals for model assumptions and using the Hosmer and Lemeshow goodness-of-fit test. ORs (or HRs), 95\% CIs and $\mathrm{p}$ values will be reported.

For the validation of the Bristol Stool Chart and Bliss Stool Classification System we will use the WHO definition of diarrhoea as the reference standard. We will compute agreement between the WHO definition of diarrhoea and the Bristol Stool Chart first; and then the Bliss Stool Classification System diarrhoea, using the kappa statistic.

All analyses will be performed with SPSS software (V.22.0, 2013). ${ }^{24}$

\section{Ethics and dissemination}

We have received local research ethics approval for the DICE-ICU Study in all participating centres. There are no safety concerns for enrolled patients. Information privacy will be addressed by de-identified data that is stored in password-protected computers in locked research offices at each centre. There is a waiver of informed consent for this observational, non-interventional study in all centres except one that required written informed consent.

The results of this study will be disseminated by presenting the findings locally at each participating hospital, as well as nationally and internationally at critical care and gastroenterology conferences. Findings will be shared with interested national societies crafting guidelines in critical care. We will publish the results in a peer-review journal.

\section{DISCUSSION}

On daily ICU rounds, diarrhoea is discussed and addressed by a multidisciplinary team of clinicians - nurses, physicians, pharmacists and dietitians - and sometimes by concerned family members. Better understanding of the prevalence, characterisation, risk factors and consequences of diarrhoea will inform patient care for each of these professionals. Strategies initiated by each group could be implemented to prevent or treat diarrhoea, in turn decreasing complications such as skin breakdown, electrolyte abnormalities and nutritional deficiencies. For example, pharmacists may suggest changing medications, dietitians may modify feeding solutions, nurses may insert faecal management devices, families and bedside clinicians may increase the use of protective materials and devices when entering the patient's room. Clinicians need to understand whether these interventions provide any benefit, cause any harm, and whether their cost is justified by the expected consequences associated with their use.

The burden of illness of diarrhoea for patients appears to vary based on the definition used, highlighting the importance of making the definition explicit when citing incidence rates. The DICE-ICU study will employ the three simplest measurement tools for diarrhoea that are candidates for use in the busy ICU setting (the WHO definition, Bristol Stool Chart and Bliss Stool Classification System). Clinicians perceive a high burden of illness and workload associated with diarrhoea in the ICU. ${ }^{25}$ Patients with diarrhoea often have extensive work-ups to identify the underlying aetiology of diarrhoea. The European Society of Intensive Care Medicine Working Group on Abdominal Problems (2012) emphasises that more research is required to identify the mechanism of diarrhoea in critically ill patients, to identify different phenotypes of diarrhoea, and thus, potential therapies. ${ }^{26}$

A mechanism of interest for diarrhoea includes alteration in the gut microbiota during critical illness. A study by Iapichino and colleagues ${ }^{27} 28$ demonstrated in 15 critically ill patients who had not been exposed to antibiotics or steroids prior to ICU admission had a reduction in intestinal anaerobes with an increase in Enterococcus isolates. Interestingly, 12 of the 15 patients developed diarrhoea and were also found to be negative for Clostridium difficile infection (CDI) ${ }^{27} \mathrm{~A}$ recent pilot prospective cohort study examined changes in faecal microbiota in 34 septic and non-septic critically ill patients in centres where systematic decontamination of the digestive tract is used compared with 15 healthy controls. ${ }^{29}$ The authors found low diversity of species in the critically ill patient cohort compared with healthy controls including loss of Faecalibacterium, Pseudobutyrivibrio, Ruminococcus, Subdoligranulum. ${ }^{29}$ There was also an increase of $>75 \%$ of one genus in four of the 34 patients with Enterococcus, Staphylococcus, Escherichia and Shigella in the critically ill patients which was not seen in the healthy controls cohort. ${ }^{29}$ In this cohort there was no CDI; however, it is unclear if these patients had diarrhoea during admission. These studies highlight the importance of future research into the microbiota during critical illness and how this may influence a patient's propensity to develop diarrhoea.

While many reasons for diarrhoea exist in the ICU, infectious aetiologies are of particular concern. Although CDAD is a common concern, only a small percentage $(11 \%)$ of patients with diarrhoea are found to have CDAD. ${ }^{30}$ The prevalence of CDAD in ICU patients is approximately $2 \%$ across a variety of ICUs, based on a recent systematic review of 16 retrospective and six prospective studies. ${ }^{30}$ To date, there are only four prospective cohort studies focusing on ICU-acquired CDAD describing 92 patients. ${ }^{31-34}$ Patients with ICU-acquired CDAD appear to have an increased length of ICU and hospital stay compared with patients without CDAD. ${ }^{35}$ However, this lack of high quality, observational data establishing the prevalence of ICU-acquired CDAD can lead to over-investigation, over-treatment and over-attribution of diarrhoea to this infection, potentially delaying the diagnosis of the true aetiology of diarrhoea. DICE-ICU will contribute to the growing knowledge of the prevalence of ICU-acquired CDAD. Data generated on the probability of various other aetiologies of diarrhoea in the DICE-ICU study will offer probabilities associated with each differential diagnosis, and may help to 
rationalise common, sometimes unnecessary resource-intensive investigations when seeking the root cause of diarrhoea.

A more detailed understanding of diarrhoea in the ICU will also help to refine approaches to care for patients with this problem. Future research may also illuminate whether diarrhoea decreases physical contact with patients (eg, pre-emptive isolation of patients associated with less frequent examination by physicians, and shorter visits by clinicians and families).

DICE-ICU has several strengths. It is a large prospective cohort study that encompasses both academical and community ICUs around the world. The study population will reflect a broad cohort of patients, enhancing the generalisability of the results. The sample size and enrolment of heterogeneous patients will also allow for detailed examination of the incidence, risk factors and consequences of diarrhoea, and will provide the first prospective study of incident cases of ICU acquired CDAD, examining the associated illness severity in this setting.

DICE-ICU has some potential limitations. Incomplete bedside documentation of bowel movements could introduce missing data; reporting bias may influence other data collection. Given the design, observer bias might influence some practices recorded as consequences of diarrhoea. Although prospective cohort studies allow for identification of risk factors, there is the potential to identify spurious associations. Infants and children are excluded from this investigation.

The DICE-ICU study will generate current, detailed multicentre clinical evidence on a common condition affecting many critically ill patients and influencing different healthcare professionals in the ICU setting. As an international investigation, it will also be the largest prospective study to examine the frequency of, predisposing factors for and consequences of diarrhoea to inform critical care practice and future research.

\section{Author affiliations}

${ }^{1}$ Department of Health Research Methods, Evidence and Impact, McMaster University, Hamilton, Ontario, Canada

${ }^{2}$ Division of Gastroenterology, Department of Medicine, McMaster University, Hamilton, Ontario, Canada

${ }^{3}$ Department of Medicine, McMaster University, Hamilton, Ontario, Canada ${ }^{4}$ Division of Critical Care, Department of Medicine, McMaster University, Hamilton, Ontario, Canada

${ }^{5}$ Division of Critical Care Medicine, Department of Medicine, Niagara Health System, Saint Catharines Site, St. Catharines, Ontario, Canada

${ }^{6}$ School of Pharamcy, Northeastern University, Bouve College of Health Sciences, Boston, Massachusetts, USA

${ }^{7}$ Farncombe Family Digestive Health Research Institute, McMaster University, Hamilton, Ontario, Canada

${ }^{8}$ Department of Critical Care, Mayo Clinic, Scottsdale, Arizona, USA

${ }^{9}$ Department of Critical Care Medicine, Kingston General Hospital, Kingston, Ontario, Canada

${ }^{10}$ Department of Critical Care Medicine, Faculty of Health Sciences, Queen's University, Kingston, Ontario, Canada

${ }^{11}$ Department of Emergency and Critical Care, Imam Abdulrahman Bin Faisal University, King Fahd Hospital of the University, Al Khobar, Saudi Arabia
Acknowledgements Thank you to Ms. Suzanne Duschesne and Ms. Lisa Buckingham for their assistance in database creation and data management.

Contributors Conception and design: JCD, DJC, WA, LM. Acquisition, analysis and interpretation of the data: JCD, DJC, WA, LM, KS, JWD, MD, EHD, PM, DA, LT, JM, JLYT, RJ, AT, CH, TK, RC-C, MSSA. Analysis of the data: JCD, LM, DJC. Drafting the manuscript: JCD, DJC, LM, LT, EHD, JWD, MD, JM, JLYT . Critiquing the manuscript: JCD, DJC, WA, LM, KS, JWD, MD, EHD, PM, DA, LT, JM, JLYT, RJ, AT, CH, TK, RC-C, MSSA. Final approval: All authors provided final approval of the manuscript.

Funding This work was supported by peer-review grants from the Hamilton Regional Medical Associates, Physicians Services Incorporated of Ontario, Canadian Association of Gastroenterology and Hamilton Health Sciences Department of Medicine (Dr. J. Dionne) and the Canadian Institutes for Health Research (Dr. D. Cook).

Competing interests None declared.

Patient consent for publication Not required.

Ethics approval Yes

Provenance and peer review Not commissioned; externally peer reviewed.

Open access This is an open access article distributed in accordance with the Creative Commons Attribution Non Commercial (CC BY-NC 4.0) license, which permits others to distribute, remix, adapt, build upon this work non-commercially, and license their derivative works on different terms, provided the original work is properly cited, appropriate credit is given, any changes made indicated, and the use is non-commercial. See: http://creativecommons.org/licenses/by-nc/4.0/.

\section{REFERENCES}

1. Whelan K, Judd PA, Taylor MA. Assessment of fecal output in patients receiving enteral tube feeding: validation of a novel chart. Eur J Clin Nutr 2004;58:1030-7.

2. Thibault R, Graf S, Clerc A, et al. Diarrhoea in the ICU: respective contribution of feeding and antibiotics. Crit Care 2013;17:R153-8.

3. Schiller LR, Pardi DS, Spiller R, et al. Gastro 2013 APDW/WCOG Shanghai working party report: chronic diarrhea: definition, classification, diagnosis. J Gastroenterol Hepatol 2014;29:6-25.

4. WHO. World Health Organization definition of diarrhea.. 2016 http:// www.who.int/topics/diarrhoea/en/.

5. Lewis SJ, Heaton KW. Stool form scale as a useful guide to intestinal transit time. Scand J Gastroenterol 1997;32:920-4.

6. Mearin F, Lacy BE, Chang L, et al. Bowel Disorders. Gastroenterology 2016. doi: 10.1053/j.gastro.2016.02.031. [Epub ahead of print 18 Feb 2018].

7. Caroff DA, Edelstein PH, Hamilton K, et al. The Bristol stool scale and its relationship to Clostridium difficile infection. J Clin Microbiol 2014;52:3437-9.

8. Zimmaro Bliss D, Larson SJ, Burr JK, et al. Reliability of a stool consistency classification system. J Wound Ostomy Continence Nurs 2001;28:305-13.

9. Zimmaro Bliss D, Dhamani KA, Savik K, et al. Tool to classify stool consistency: content validity and use by persons of diverse cultures. Nurs Health Sci 2003;5:115-21.

10. Jack L, Coyer F, Courtney M, et al. Diarrhoea risk factors in enterally tube fed critically ill patients: a retrospective audit. Intensive Crit Care Nurs 2010;26:327-34.

11. Jack L, Coyer F, Courtney M, et al. Probiotics and diarrhoea management in enterally tube fed critically ill patients--what is the evidence? Intensive Crit Care Nurs 2010;26:314-26.

12. McClave SA, Taylor BE, Martindale RG, et al. Guidelines for the Provision and Assessment of Nutrition Support Therapy in the Adult Critically III Patient: Society of Critical Care Medicine (SCCM) and American Society for Parenteral and Enteral Nutrition (A.S.P.E.N.). JPEN J Parenter Enteral Nutr 2016;40:159-211.

13. Reintam A, Parm P, Redlich U, et al. Gastrointestinal failure in intensive care: a retrospective clinical study in three different intensive care units in Germany and Estonia. BMC Gastroenterol 2006;6:19.

14. Marcon AP, Gamba MA, Vianna LA. Nosocomial diarrhea in the intensive care unit. Braz J Infect Dis 2006;10:384-9.

15. Tirlapur N, Puthucheary ZA, Cooper JA, et al. Diarrhoea in the critically ill is common, associated with poor outcome, and rarely due to Clostridium difficile. Sci Rep 2016;6:24691.

16. Oczkowski SJW, Duan EH, Groen A, et al. The Use of Bowel Protocols in Critically III Adult Patients: A Systematic Review and Meta-Analysis. Crit Care Med 2017;45:e718-e726. 
17. Dionne JC, Sullivan K, Mbuagbaw L, et a/Tu1068 Diarrhea in the Intensive Care Unit: Epidemiology (The DICE Study). Gastroenterology 2016;150:S832.

18. iDatafax Clinical Systems Incorporated. iDatafax Clinical Systems Incorporated, version 4.3.0. Seattle, WA: DF/NET Research INC, 2013.

19. McDonald LC, Gerding DN, Johnson S, et al. Clinical Practice Guidelines for Clostridium difficile Infection in Adults and Children: 2017 Update by the Infectious Diseases Society of America (IDSA) and Society for Healthcare Epidemiology of America (SHEA). Clin Infect Dis 2018;66:e1-e48.

20. Crobach MJ, Planche T, Eckert C, et al. European Society of Clinical Microbiology and Infectious Diseases: update of the diagnostic guidance document for Clostridium difficile infection. Clin Microbiol Infect 2016;22(Suppl 4):S63-S81.

21. Surawicz CM, Brandt LJ, Binion DG, et al. Guidelines for diagnosis, treatment, and prevention of Clostridium difficile infections. Am J Gastroenterol 2013;108:478-98. quiz 499.

22. Ogundimu EO, Altman DG, Collins GS. Adequate sample size for developing prediction models is not simply related to events per variable. J Clin Epidemiol 2016;76:175-82.

23. Faul F, Erdfelder E, Lang AG, et al. G*Power 3: a flexible statistical power analysis program for the social, behavioral, and biomedical sciences. Behav Res Methods 2007;39:175-91.

24. Hosmer DW, Lemeshow S. Applied Logistic Regression. New York: Wiley IBM Corp, 2013. IBM SPSS Statistics for Windows, Version 22.0: Armonk, NY: IBM Corp.

25. Heidegger CP, Graf S, Perneger T, et al. The burden of diarrhea in the intensive care unit (ICU-BD). A survey and observational study of the caregivers' opinions and workload. Int J Nurs Stud 2016;59:163-8.

26. Reintam Blaser A, Malbrain ML, Starkopf J, et al. Gastrointestinal function in intensive care patients: terminology, definitions and management. Recommendations of the ESICM Working Group on Abdominal Problems. Intensive Care Med 2012;38:384-94.

27. Iapichino G, Callegari ML, Marzorati S, et al. Impact of antibiotics on the gut microbiota of critically ill patients. J Med Microbiol 2008;57(Pt 8):1007-14.

28. lapichino G, Lankelma JM, Joost Wiersinga W. Gut microbiota disruption in critically ill patients : Discussion on "Critically ill patients demonstrate large interpersonal variation of intestinal microbiota dysregulation: a pilot study". Intensive Care Med 2017;43:718-9.

29. Lankelma JM, van Vught LA, Belzer C, et al. Critically ill patients demonstrate large interpersonal variation in intestinal microbiota dysregulation: a pilot study. Intensive Care Med 2017;43:59-68.

30. Karanika S, et al. Prevalence and Clinical Outcomes of Clostridium difficile Infection in the Intensive Care Unit: A Systematic Review and Meta-Analysis. Open Forum Infect Dis 2016;3(1:ofv186.

31. Rotimi VO, Mokaddas EM, Jamal WY, et al. Hospital-acquired Clostridium difficile infection amongst ICU and burn patients in Kuwait. Med Princ Pract 2002;11:23-8.

32. Zahar JR, Schwebel C, Adrie C, et al. Outcome of ICU patients with Clostridium difficile infection. Crit Care 2012;16:R215.

33. Lumpkins K, Bochicchio GV, Joshi M, et al. Clostridium difficile infection in critically injured trauma patients. Surg Infect 2008;9:497-501.

34. Nguyen NQ, Ching K, Fraser RJ, et al. Risk of Clostridium difficile diarrhoea in critically ill patients treated with erythromycin-based prokinetic therapy for feed intolerance. Intensive Care Med 2008;34:169-73.

35. Dodek PM, Norena M, Ayas NT, et al. Length of stay and mortality due to Clostridium difficile infection acquired in the intensive care unit. J Crit Care 2013;28:335-40. 